\title{
Equivalence of Extrinsic and Intrinsic Quantization for Observables not Preserving the Vertical Polarization
}

\author{
C. Emmrich \\ Fakultät für Physik der Universität Freiburg, Hermann-Herder-Str. 3, D-7800 Freiburg, FRG
}

Received January 24, 1992

\begin{abstract}
The equivalence of Dirac quantization and intrinsic quantization for arbitrary observables not preserving the vertical polarization is examined for systems with first class constraints that may be considered as the vanishing of the momentum map to a lifted group action. Using a generalized Weyl ordering prescription applicable to arbitrary cotangent bundles we derive necessary and sufficient conditions for the equivalence of the two approaches for different classes of functions. A strong obstruction is found if one requires equivalence for all invariant functions, essentially only admitting trivial bundles. By a restriction to an adequate class of "strongly admissible functions", equivalence can always be obtained in the case of a free group action. Implications for the case of non-free actions and the dependence on the particular quantization scheme are discussed.
\end{abstract}

\section{Introduction}

Systems with first class constraints can be quantized in essentially two conceptually different ways: One may impose the constraints classically, divide out the gauge transformations generated by the constraints and quantize the resulting unconstrained system ("intrinsic quantization"). On the other hand, one may try to quantize the original system without the constraints and then impose the constraints as conditions on the physical states ("extrinsic quantization," in particular Dirac quantization [4]).

Normally, first class constraints arise as a consequence of "redundancy symmetries" of the Lagrangian of the theory, i.e., the presence of time-dependent symmetry transformations which do not relate different physical states but different redundant descriptions of the same state (as in gauge theories, where true physical states correspond to gauge equivalence classes of gauge potentials). If this symmetry is supposed to be a redundancy symmetry on the quantum mechanical level as well, the intrinsic method is conceptually preferable. On the other hand, the extrinsic quantization scheme is often preferable from a practical, computational 
point of view: First, the structure of extended configuration and phase space is often much simpler than that of the reduced spaces, which generally are not even manifolds [7]. Secondly, it is often easier in relativistic theories to maintain manifest Lorentz covariance in the extrinsic formalism, as for practical computations in the intrinsic scheme one usually chooses a gauge fixing, i.e., one locally embeds the reduced space, consisting of equivalence classes of redundant states related by redundancy transformations, into the extrinsic space.

Thus, the question arises whether extrinsic and intrinsic quantization are equivalent. As the more elaborate quantization scheme of BRST quantization is known to be equivalent to Dirac quantization for a wide class of systems [6], this question is of particular importance. It has two aspects: First, one has to find a natural isomorphism of the Hilbert spaces, and secondly, one has to check whether this isomorphism intertwines the operators corresponding to the same observable ("Dirac equivalence condition for observables").

Concerning the first aspect, there exist several positive results of different generality and strength within the framework of geometric quantization using the vertical polarization on cotangent bundles for the cases when the constraints are linear in the momenta; in some cases (i.e., the cases of nonunimodular groups and structure functions instead of structure constants) some slight modifications are needed $[1,5,8,16,17]$. Especially, in the case of a compact Lie-group acting on phase space by the lifting of a free action on configuration space, the complete program of geometric quantization with its different steps (prequantum bundle, metalinear frame bundle, half-form bundle, ...) can be carried out and strict equivalence may be shown [8], whereas the analysis in the more general cases is usually restricted to the study of half-densities corresponding to a trivial prequantum line bundle.

Concerning the second aspect, equivalence results are established in the articles mentioned above for a very restricted class of observables, namely for those which preserve the vertical polarization, i.e., which are at most linear in the momenta, the restriction occurring due to the problems of geometric quantization in the quantization of not-polarization-preserving functions. On the other hand, for particular examples and for special quadratic observables the equivalence is known not to hold in general, or to need some additional care and adapted quantization schemes $[10,13,14]$. Since in most applications the Hamiltonian is quadratic in the momenta the equivalence question for observables which do not preserve the vertical polarization is of particular importance.

In this article we are going to examine the equivalence question for observables which are arbitrary polynomials in the momenta. The main problem for such an examination is the need for a definite quantization scheme which assigns to arbitrary functions (or at least a sufficiently large class of functions) operators on the respective Hilbert spaces. Within the framework of geometric quantization, the only existing procedure for functions not preserving the polarization is the use of BKS-kernels [15]. This scheme is much less stringent than the quantization method for polarization preserving functions and has two major disadvantages: First, some rather complicated regularity conditions have to be fulfilled, and secondly, there is in general no guarantee that the resulting operators are even formally selfadjoint.

For these reasons we shall use a special ansatz for the quantization of systems whose phase spaces are cotangent bundles. This ansatz, which is due to Underhill [18], allows the quantization of arbitrary observables which are polynomial in the 
momenta, yielding quantum operators which are differential operators of the order of the polynomial and which are guaranteed to be at least formally selfadjoint. (A generalization of this ansatz to more general orderings as well as the connection to the methods of $[19,20]$ using the symbol calculus for pseudodifferential operators is discussed in [12].) Furthermore, this quantization scheme guarantees that the sum of two classical observables is quantized by the sum of the corresponding operators, which is not true for the BKS-kernel procedure even in those cases in which it yields well defined formally selfadjoint operators.

In order to concentrate on the equivalence problem for observables and not to overload the exposition with additional technical problems we shall restrict ourselves to the situation where the first aspect of the equivalence problem, that of a natural isomorphism of the respective Hilbert spaces, is particularly simple, namely the case of lifted free group actions of a compact connected Lie-group $\mathbf{G}$ of dimension $r$ on a cotangent bundle $T^{*} P$. In this case, the constraints have the form of the vanishing of the corresponding canonical momentum map $\mathscr{J}$.

In this situation, the following theorem guarantees that the reduced phase space is a cotangent bundle again, with the reduced configuration space $Q=P / \mathbf{G}[11,8]$ :

Theorem 1. Let a Lie-group $\mathbf{G}$ act properly and freely on a manifold $P$, and let $\mathscr{J}$ denote the canonical momentum mapping to the lifted group action on $T^{*} P$, i.e., $\mathscr{J}$ : $T^{*} P \rightarrow \mathscr{G}^{*}, \alpha_{q} \mapsto \mathscr{J}\left(\alpha_{q}\right)$ with $\left\langle\mathscr{J}\left(\alpha_{q}\right), A\right\rangle=\alpha_{q}\left(A^{*}(q)\right)$ for $A \in \mathscr{G}$, where $A *$ denotes the fundamental vector field of $A \in \mathscr{G}$. Then there is a canonical isomorphism:

$$
\mathscr{J}^{-1}(0) / \mathbf{G} \cong T^{*}(P / \mathbf{G})
$$

Via this isomorphism any weakly $\mathbf{G}$-invariant function on $T^{*} P$, i.e., any $\phi \in \mathscr{F}\left(T^{*} P\right)$ with $\left.\left(R_{g}^{*} \phi-\phi\right)\right|_{\mathscr{J}^{-1}(0)} \equiv 0$, corresponds to $\underset{\tilde{\phi}}{\text { function }}$ on $T^{*}(P / \mathbf{G})$, whereas the function on $T^{*} P$ corresponding to a given $\tilde{\phi} \in \mathscr{F}\left(T^{*}(P / \mathbf{G})\right)$ is only determined up to the addition of a function in $\langle\mathscr{J}\rangle$, the ideal in $\mathscr{F}\left(T^{*} P\right)$ generated by the components of the momentum mapping. In more mathematical terms this means that for a free group action (or more generally, if 0 is a weakly regular value of $\mathscr{J}$ ) there is an isomorphism

$$
\mathscr{F}\left(T^{*}(P / \mathbf{G})\right) \cong\left(\mathscr{F}\left(T^{*} P\right) /\langle\mathscr{J}\rangle\right)_{\mathbf{G}},
$$

where we denote by $\mathscr{A}_{\mathbf{G}}$ the $\mathbf{G}$-invariant elements in the algebra $\mathscr{A}$. For a compact group any weakly invariant function can be made invariant without changing its values on the constraint surface by averaging over the group with the invariant Haar-measure. Hence, we get an isomorphism:

$$
\mathscr{F}\left(T^{*}(P / \mathbf{G})\right) \cong \mathscr{F}\left(T^{*} P\right)_{\mathbf{G}} /\langle\mathscr{J}\rangle_{\mathbf{G}} .
$$

In any case, there is a whole class of functions on extended phase space corresponding to the same function on reduced phase space. Thus, it will be necessary to fix the class of admissible functions on $T^{*} P$ corresponding to the same observable on $T^{*}(P / \mathbf{G})$.

In the following section we shall first present the generalized Weyl ordering prescription on arbitrary cotangent bundles developed in [18] and a modification of it particularly suitable for our purposes. In Sect. 3 we shall describe the extrinsic and intrinsic Hilbert spaces and give a very explicit representation of the natural isomorphism between them. In the next section we shall finally prove some 
necessary and sufficient conditions for the validity of the Dirac equivalence condition for different classes of polynomial functions for our modified Weyl ordering prescription. In Sect. 5 we shall study the same problem for the unmodified quantization scheme, and in Sect. 6 we shall eventually make some conclusions and remarks on the dependence on the particular quantization scheme.

\section{Quantization via Generalized Weyl Ordering}

The idea of this quantization method is to generalize the integral representation for the Weyl ordering to arbitrary cotangent bundles: On $T^{*} \mathbb{R}^{n}=\mathbb{R}^{2 n}$, the matrix element of the operator corresponding to an observable $f(q, p) \in \mathscr{F}\left(T^{*} \mathbb{R}^{n}\right)$ may be expressed as [18]:

$$
\begin{aligned}
\langle\phi, \mathscr{Q}(f) \psi\rangle=\pi^{-n} \iint \overline{\phi(x-v)} \psi(x+v) e^{-2 i\langle p . v\rangle} d v^{1} \ldots d v^{n} \\
\\
\quad f(x, p) d x^{1} \ldots d x^{n} d p_{1} \ldots d p_{n} .
\end{aligned}
$$

While the measure $d x^{1} \ldots d x^{n} d p_{1} \ldots d p_{n}$ is the Liouville measure on phase space, which is canonically defined on any cotangent bundle, the first integration does a priori not make any sense on an arbitrary cotangent bundle. However, if one chooses an arbitrary connection on the configuration space $Q$, it may be identified locally, in a suitable neighbourhood of any point $q \in Q$, with an open ball in $T_{q} Q$, and the integration can be done over $T_{q} Q$.

More explicitly, the Hilbert space $\mathbf{H}$ of states for a system with orientable configuration space can be constructed as the space of half-densities with finite norm. Choosing a connection on $Q$, one may, for any $q \in Q$, pull back any halfdensity with support in a sufficiently small neighbourhood of $q$ to $T_{q} Q$ with the exponential mapping. Hence, setting $I_{q}: T_{q} Q \rightarrow T_{q} Q, v_{q} \mapsto-v_{q}$, the expression $\chi\left(q, v_{q}\right)\left(\exp ^{*} \psi\right)\left(\left(\exp \circ I_{q}\right)^{*} \phi\right)$ is a well defined density on $T_{q} Q$ for a suitable cutoff function $\chi$ and any half-densities $\phi, \psi$, and we may set

$$
\Phi\left(\alpha_{q}\right):=\int e^{-2 i x_{q}\left(v_{q}\right)} \chi\left(q, v_{q}\right)\left(\exp ^{*} \psi\right) \overline{\left(\left(\exp \circ I_{q}\right)^{*} \phi\right)},
$$

yielding a well defined function on $T^{*} Q$.

Therefore, the matrix element $\langle\phi, \mathcal{Q}(f) \psi\rangle$ may be defined as:

$$
\langle\phi, \mathscr{Z}(f) \psi\rangle:=\pi^{-n} \int \Phi\left(\alpha_{q}\right) f\left(\alpha_{q}\right) d \Omega,
$$

where $d \Omega$ denotes the Liouville measure on $T^{*} Q$. In [18], it is shown that $2(f)$ is a well defined partial differential operator of order degree $(f)$ for any $f$ polynomial in the momenta, independent of the particular choice of the cutoff function $\%$.

For an observable which is a polynomial in the momenta a rather explicit coordinate representation of the matrix elements of the corresponding operator can be derived: Denoting local coordinates in a neighbourhood of $q \in Q$ by $\left(x^{l}\right)_{l=1} \ldots n$ and the induced coordinates on $T_{q} Q$ by $\left(v^{l}\right)_{l=1 \ldots n}$, (6) yields for $f=T^{i_{1} \cdots \iota_{m}}(x) p_{i_{1}} \ldots p_{i_{m}}$ :

$$
\langle\phi, 2(f) \psi\rangle=\left.(2 i)^{-m} \int d x^{1} \ldots d x^{n} T^{\iota_{1} \ldots i_{m}}(x) \frac{\partial^{m}}{\partial v^{i_{1}} \ldots \partial v^{i_{m}}} D(x, v)\right|_{v=0},
$$

where $D(x, v) d v^{1} \ldots d v^{n}$ is the local expression of $\%\left(q, v_{q}\right)\left(\exp ^{*} \psi\right) \overline{\left.\left(\exp ^{\circ} I_{q}\right)^{*} \phi\right)}$. 
For the special class of observables compatible with the vertical polarization, namely those at most linear in the momenta, which are of the form $f\left(\alpha_{q}\right)=\alpha_{q}\left(X_{q}\right)+g(q)$ for a vector field $X \in \mathscr{X}(Q)$, this quantization scheme yields the same standard expression as geometric quantization using the trivial line bundle:

$$
\mathscr{Z}(f) \phi=\left\{-i \mathbf{L}_{X}+g(q)\right\} \phi .
$$

Here, $\mathbf{L}_{X} \phi$ denotes the Lie-derivative of the half-density $\phi$.

For an observable $f(x, p)=T^{i j}(x) p_{i} p_{j}$ quadratic in the momenta, the quantization scheme yields:

$$
\mathscr{D}(f)=-\left[T^{\iota \jmath} \nabla_{i} \nabla_{j}+\left(\nabla_{i} T^{i j}\right) \nabla_{j}+\frac{1}{4}\left(\nabla_{i} \nabla_{j} T^{i j}\right)+\frac{1}{12} R_{i j} T^{i j}\right] .
$$

In particular, if $\nabla$ is the Levi-Civita connection of a Riemannian metric $\mathbf{g}, \mathbf{g}^{i j}(x) p_{i} p_{j}$ is quantized by $-\Delta-\frac{1}{12} R$, where $R$ is the scalar curvature.

The quantization ansatz presented above only depends on the choice of a connection, not of a metric on the configuration space. (Without loss of generality, the connection may be chosen torsion free as only the exponential map plays a role in the whole construction.) However, due to the curvature terms arising by the half-density character of $\phi, \psi$ in (6), this scheme will prove not to be particularly adapted to our purposes. If the covariant derivative $\nabla$ on $Q$ is the Levi-Civita connection to a Riemannian metric $\mathbf{g}$, the quantization scheme may be suitably modified without destroying the formal selfadjointness of the operators and without changing the operators assigned to classical functions that are at most linear in the momenta:

The metric $\mathbf{g}$ canonically induces a measure $\mu$ which in local coordinates $\left(x_{i}\right)$ can be expressed as $\mu=\sqrt{\operatorname{det}\left(g_{i j}\right)(x)} d x_{1} \ldots d x_{n}$. This measure induces an identification of the Hilbert space of half-densities with finite norm with the Hilbert space $\hat{\mathbf{H}}:=L^{2}(Q, \mu)$ of functions on $Q$ which are square integrable with respect to $\mu$ : Any half density with finite norm may be written as $\phi=\hat{\phi}|\mu|^{\frac{1}{2}}$ for a square integrable function $\hat{\phi}$.

The Riemannian metric $\mathbf{g}$ on $Q$ defines a Euclidian metric and hence a unique canonical measure on any fibre $T_{q} Q$, which we shall denote by $d^{n} v(q)$. In local coordinates $\left(x^{i}\right)$ on $Q$ and induced coordinates $\left(v^{i}\right)$ on $T_{q} Q$, this measure has the form $\sqrt{\operatorname{det}\left(g_{i j}(x)\right)} d v^{1} \ldots d v^{n}$, where $g_{i j} d x^{i} d x^{j}$ is the local expression of the Riemannian metric. (In general, $d^{n} v(q)$ being translation invariant, this measure does not coincide with the pullback of the measure $\mu$ via the exponential mapping.)

We may now define

$$
\widehat{\Phi\left(\alpha_{q}\right)}:=\int e^{-2 i \alpha_{q}\left(v_{q}\right)} \chi\left(q, v_{q}\right)(\exp * \hat{\psi}) \overline{\left(\left(\exp \circ I_{q}\right)^{*} \hat{\phi}\right)} d^{n} v(q)
$$

and the matrix element of the operator $\hat{\mathcal{Q}}(f)$ by (6) with $\Phi$ replaced by $\hat{\Phi}$, yielding for $f=T^{i_{1} \ldots i_{m}}(x) p_{\imath_{1}} \ldots p_{i_{m}}$ the coordinate expression:

$$
\langle\phi, \hat{\mathscr{Q}}(f) \psi\rangle=\left.(2 i)^{-n} \int \sqrt{\operatorname{det}\left(g_{i j}(x)\right)} d x^{1} \ldots d x^{n} T^{i_{\imath} \ldots i_{m}} \frac{\partial^{m}}{\partial v^{i_{1}} \ldots \partial v^{i_{m}}} D \widehat{(x, v)}\right|_{v=0},
$$


where $D \widehat{(x, v)}=\chi\left(x, v_{x}\right)\left(\exp _{x}^{*} \hat{\psi}\right) \overline{\left(\left(\exp _{x} \circ I_{q}\right)^{*} \hat{\phi}\right)}$. This modified quantization ansatz assigns to $f=T^{i j} p_{\iota} p_{j}$ the operator

$$
\hat{\mathcal{Z}}(f)=-\left[T^{i j} \nabla_{i} \nabla_{j}+\left(\nabla_{i} T^{i j}\right) \nabla_{j}+\frac{1}{4}\left(\nabla_{i} \nabla_{j} T^{i j}\right)\right]
$$

on $\hat{\mathbf{H}}:=L^{2}(Q, \mu)$. In particular, $g^{i j} p_{i} p_{j}$ is quantized by the Laplace-operator to the metric $\mathbf{g}$.

\section{Dirac Quantization on Principal Bundles}

In the following we assume that a compact connected Lie-group $\mathbf{G}$ acts freely and properly on the extrinsic configuration space $P$, i.e., the extrinsic configuration space $P$ is a $\mathbf{G}$-principal bundle over $Q:=P / \mathbf{G}$ with projection $\pi$. In order to obtain the usual sign conventions we assume that $\mathbf{G}$ is acting from the right. (Using the inverse map $g \mapsto g^{-1}$ on $\mathbf{G}$, this can be easily translated to a left action.) For simplicity, we shall assume that $Q$ (and hence $P$ ) is orientable. We shall use the notation $R_{g} q=q \cdot g$. Furthermore we choose a Riemannian metric $\mathbf{g}$ on $P$ with corresponding Levi-Civita connection $\nabla$ to define the extrinsic quantization, i.e.,

$$
\begin{aligned}
2 \mathbf{g}\left(\nabla_{X} Y, Z\right)= & X \mathbf{g}(Y, Z)+Y \mathbf{g}(X, Z)-Z \mathbf{g}(X, Y) \\
& +\mathbf{g}([X, Y], Z)+\mathbf{g}([Z, X], Y)+\mathbf{g}(X,[Z, Y]) .
\end{aligned}
$$

As we want $\mathbf{G}$-invariant functions to be quantized by $\mathbf{G}$-invariant operators, we assume that $\mathbf{g}$ is $\mathbf{G}$-invariant. Now we have the following well known lemma $[2,3]$ :

Lemma 2. There is a one-to-one correspondence between right $\mathbf{G}$-invariant Riemannian metrics $\mathbf{g}$ on $P$ and triples $(\tilde{\mathbf{g}}, \alpha, \mathbf{h})$, where $\tilde{\mathbf{g}}$ is a metric on $Q=P / \mathbf{G}, \alpha$ is a connection form on $P$ as a principal $\mathbf{G}$-bundle over $Q$ and $\mathbf{h}$ is a smooth mapping assigning to any $q \in Q$ an invariant metric $\mathbf{h}(q)$ on the orbit $\pi^{-1}(q)$.

The connection on $P$ is constructed by defining the subbundle of TP consisting of vectors orthogonal to the orbits as the horizontal distribution corresponding to $\alpha$. $\tilde{\mathbf{g}}$ is defined by $\tilde{\mathbf{g}}(\tilde{X}(\tilde{q}), \tilde{Y}(\tilde{q}))=\mathbf{g}\left(\tilde{X}^{h}(q), \tilde{Y}^{h}(q)\right)$ for some $q \in \pi^{-1}(\tilde{q})$, where $\tilde{X}^{h}$ denotes the horizontal lift of $\tilde{X}$. For $q \in P$ we denote by $\mathscr{H}_{q}$ the horizontal space at $q$, and for a vector field $X$ by $\mathscr{H}(X)$ its horizontal projection.

A particular simple choice for the function $\mathbf{h}$ is a "constant" metric in that sense, that one chooses a $\mathbf{G}$-invariant metric on $\mathbf{G}$ and defines the corresponding metrics on the orbits by a local trivialization of $P$. It is easy to check that the function $\mathbf{h}$ defined in this way is independent of the trivialization because of the invariance of the metric on $\mathbf{G}$.

The orbits are totally geodesic iff $\mathbf{g}(q)\left(\nabla_{A^{*}} B^{*}(q), \tilde{Z}^{h}(q)\right)=0$ for arbitrary $q \in P$, $A, B \in \mathscr{G}$ and $\tilde{Z} \in \mathscr{X}(Q)$. Using (13) one gets the equivalent condition

$$
\left(\tilde{Z}^{h} \mathbf{g}\left(A^{*}, B^{*}\right)\right)(q)=0 .
$$

Choosing a trivialization such that the coordinate vector fields of the coordinates of the base manifold are horizontal in the point $q$, one easily sees that this condition is equivalent to the constancy of $\mathbf{h}$ in the sense above. 
The $\mathbf{G}$-invariance of the metric $\mathbf{g}$ implies the $\mathbf{G}$-invariance of the measure $\mu$, yielding:

$$
\left(\operatorname{div} A^{*}\right) \mu=L_{A^{*}} \mu=\left.\frac{d}{d t} \exp (t A)^{*} \mu\right|_{t=0}=0 .
$$

Hence, the components of the momentum map $\mathscr{J}_{A}\left(\alpha_{q}\right)=\alpha_{q}\left(A^{*}\right)$ are quantized in both quantization schemes presented in the preceding section by

$$
\mathscr{2}\left(\mathscr{J}_{A}\right)\left(\hat{\phi}|\mu|^{\frac{1}{2}}\right)=-i\left(A^{*} \hat{\phi}\right)|\mu|^{\frac{1}{2}} .
$$

Thus, the half-densities on $P$ fulfilling the Dirac condition correspond just to the G-invariant functions on $P$ :

$$
\begin{aligned}
\mathbf{H}_{\mathrm{phys}}: & =\left\{\phi \in \mathbf{H}_{\mathrm{ext}}: \mathscr{Q}\left(\mathscr{J}_{A}\right) \phi=0 \forall A \in \mathscr{G}\right\} \\
& =\left\{\hat{\phi}|\mu|^{\frac{1}{2}} \in \mathbf{H}_{\mathrm{ext}}: \hat{\phi} \in \mathscr{F}(P), R_{g}^{*} \hat{\phi}=\hat{\phi} \forall g \in \mathbf{G}\right\} .
\end{aligned}
$$

There is a canonical isomorphism of the "physical" subspace $\mathbf{H}_{\text {phys }}$ and the intrinsic Hilbert space $\mathbf{H}_{\text {int }}$ of half-densities on $Q=P / \mathbf{G}[8,16]$ : Choosing an ad-invariant scalar product on the Lie algebra $\mathscr{G}$, one may assign to any frame $\left(\tilde{v}_{[q]}^{i}\right)_{i=1 \ldots n-r}$ on $T_{[q]}(P / \mathbf{G})$ the set of frames on $T_{q} P$ which are of the form $\left(A_{1}^{*}(q), \ldots\right.$, $\left.A_{r}^{*}(q), v_{q}^{1}, \ldots, v_{q}^{n-r}\right)$, where $\left(A_{i}\right)_{i=1 \ldots r}$ is an orthonormal basis of $\mathscr{G}$ and $v_{q}^{i}$ is a vector in $T_{q} P$ projecting onto $\tilde{v}_{[q]}^{i}$. All those frames are related by linear transformations with determinant one. Hence, for an arbitrary half-density $\tilde{\phi}$ on $P / \mathbf{G}$ one may define a half-density $\phi$ on $P$ by

$$
\phi\left(A_{1}^{*}(q), \ldots, A_{r}^{*}(q), v_{q}^{1}, \ldots, v_{q}^{n-r}\right):=\tilde{\phi}\left(\tilde{v}_{[q]}^{1}, \ldots, \tilde{v}_{[q]}^{n-r}\right) .
$$

Equation (15) indeed defines a bijection from $\mathbf{H}_{\text {int }}$ to $\mathbf{H}_{\text {phys }}$, and if the group is compact and one normalizes the scalar product on $\mathscr{G}$ in such a way that the volume of the Lie group $\mathbf{G}$ with respect to the volume induced by the corresponding biinvariant measure is unity, it is even an isometry and hence an isomorphism of Hilbert spaces.

On $Q$ we define a function $\kappa$ by

$$
\kappa(\tilde{q})=\sqrt{\operatorname{det}\left(\mathbf{g}(q)\left(A_{i}^{*}(q), A_{j}^{*}(q)\right)_{i, j=1 \ldots r}\right)}
$$

for an orthonormal base $\left(A_{i}\right)_{i=1 \ldots r}$ of $\mathscr{G}$ and $q \in \pi^{-1}(\tilde{q})$. Since for a fundamental vector field $A^{*}(q \cdot g)=T_{q} R_{g}\left(\operatorname{Ad}_{g} A\right)^{*}(q)$ and $\operatorname{det}\left(\operatorname{Ad}_{g}\right)=1$ for any $g \in \mathbf{G}$ by the unimodularity of $\mathbf{G}, \kappa$ is easily seen to be a well defined function independent of the choice of $q \in \pi^{-1}(\tilde{q}) . \kappa(\tilde{q})$ obviously is just the volume of the orbit $\pi^{-1}(\tilde{q})$. With its help and the identification of half forms with functions via $|\mu|^{\frac{1}{2}}$ and $|\tilde{\mu}|^{\frac{1}{2}}$ a very explicit form of the isomorphism can be derived:

$$
U: \hat{\mathbf{H}}_{\mathrm{int}} \rightarrow \hat{\mathbf{H}}_{\mathrm{phys}} \subset \hat{\mathbf{H}}_{\mathrm{ext}}, \quad \phi_{\mathrm{int}} \mapsto \pi^{*}\left(\frac{\phi_{\mathrm{int}}}{\sqrt{\kappa}}\right) .
$$

By the theorems proved in [8] we know that this isomorphism of Hilbert spaces intertwines the operators corresponding to functions preserving the vertical polarization, i.e., to functions at most linear in the momenta, as long as one restricts oneself to $\mathbf{G}$-invariant functions on $T^{*} P$. 


\section{Equivalence for Higher Order Polynomials}

For polynomials in the momenta of order one the theorems mentioned above guarantee that the Dirac equivalence condition is satisfied independent of the choice of the representative of the equivalence class in $\mathscr{F}\left(T^{*} P\right)_{\mathbf{G}} /\langle\mathscr{J}\rangle_{\mathbf{G}}$ corresponding to a given $\tilde{\phi} \in \mathscr{F}\left(T^{*} Q\right)$. Here, the restriction to $\mathbf{G}$-invariant representatives, i.e., the use of the isomorphism (3) and not of (2) is necessary, as one may easily see by a simple example in which there are no ambiguities in the definition of Weyl quantization:

Example. Choose $P=\mathbb{R}^{n}, G=\mathbb{R}$ acting on $P$ as $\alpha \cdot\left(x_{1}, \ldots, x_{n}\right):=\left(\alpha+x_{1}\right.$, $x_{2}, \ldots, x_{n}$ ). (In order to avoid problems with normalizability and the need to use generalized eigenfunctions in a Gelfand triple, one may consider the $U(1)$-action on a torus or a cylinder equally well.) The momentum mapping to that action corresponds just to the first component $p_{1}$ of ordinary momentum $p$. The observables $x_{i}$ are quantized in the standard way as multiplication operators, $p_{i}$ as $-i \hbar \frac{\partial}{\partial x_{i}}$. Any function of the form $p_{1} f(x, p)$ vanishes on the constraint surface $p_{1}=0$, hence the operator assigned to it via Weyl ordering should vanish on the space of physical states, consisting of wave functions independent of the first variable $x_{1}$. However,

$$
\mathscr{Z}\left(p_{1} x_{1}^{2}\right)=\frac{1}{3}\left(\mathscr{2}\left(p_{1}\right) \mathscr{Q}\left(x_{1}\right)^{2}+\mathscr{2}\left(x_{1}\right) \mathscr{2}\left(p_{1}\right) \mathscr{2}\left(x_{1}\right)+\mathscr{2}\left(x_{1}\right)^{2} \mathscr{2}\left(p_{1}\right)\right),
$$

which does not vanish on the space of physical states. However, if one restricts oneself to $U(1)$-invariant representatives, i.e., if one uses the isomorphism

$$
\mathscr{F}\left(T^{*}(P / \mathbf{G})\right) \cong \mathscr{F}\left(T^{*} P\right)_{\mathbf{G}} /\langle\mathscr{J}\rangle_{\mathbf{G}},
$$

$p_{1} f(x, p)$ is only admissible if $f$ is independent of $x_{1}$. In this case, there are no ordering problems for the operators $\mathscr{Q}\left(p_{1}\right)$. They may all be moved to the right, whence the corresponding operator vanishes on the space of physical states.

As we have seen in the example, we have to restrict the class of admissible functions on phase space at least to those which are $\mathbf{G}$-invariant and not only weakly G-invariant. Theorem 4 below will show that even with this restriction there are very strong conditions on the admissible extended configuration spaces and the metric g. Using the connection form $\alpha$ it is possible to further restrict the class of admissible functions. In the rest of this section we shall prove three theorems which give necessary and sufficient conditions for the Dirac equivalence condition to hold for certain classes of functions on $T^{*} P$ :

The decomposition of $T P$ into a horizontal and a vertical subbundle induces a decomposition of $T^{*} P$ into vertical momenta, which vanish on horizontal vectors, and horizontal momenta, which vanish on vertical vectors.

Definition 1. We call a polynomial $f$ on $T^{*} P$ "horizontal" ("vertical") if it is a polynomial only in the horizontal (vertical) momenta. We call a polynomial $f$ on $T^{*} P$ strongly admissible iff it is the sum of a "horizontal" and a "vertical" polynomial.

Theorem 3. A necessary condition for the Dirac equivalence condition for strongly admissible quadratic observables to be fulfilled is that the orbits are totally geodesic submanifolds of P. In this case, the Dirac equivalence condition is satisfied for strongly 
admissible polynomials of arbitrary degree, if one uses the metric $\tilde{\mathbf{g}}$ on $Q$ induced by g for the intrinsic quantization.

Theorem 4. The Dirac equivalence condition for arbitrary invariant quadratic observables is fulfilled iff the orbits are totally geodesic submanifolds of $P$ and the connection form $\alpha$ on $P$ induced by $\mathbf{g}$ is flat. Then it is satisfied for invariant polynomials of arbitrary degree. In particular, if $Q$ is simply connected, $P$ admits a metric such that the Dirac equivalence condition is fulfilled if and only if $P$ is a trivial bundle over $Q: P \cong Q \times \mathbf{G}$.

Proof. We denote by " $\vee$ " the symmetrized tensor product. For any G-invariant vector fields $V, W \in \mathscr{X}(P)$, the polynomial $f=T^{i j} p_{i} p_{j}$ with $T=V \vee W$ is invariant. If both $V$ and $W$ are horizontal (vertical), then $f$ is "horizontal" ("vertical"), hence it is strongly admissible in both cases.

For a $\mathbf{G}$-invariant vector field $X$ and a fundamental vector field $A^{*}:\left[X, A^{*}\right]$ $=0$. Thus, $[X, \eta]$ is an invariant vertical vector field for any invariant vertical vector field $\eta$. By (13)

$$
2 \mathbf{g}\left(\nabla_{\tilde{X}^{h}} \eta, \tilde{Z}^{h}\right)=\mathbf{g}\left(\left[\tilde{Z}^{h}, \tilde{X}^{h}\right], \eta\right), 2 \mathbf{g}\left(\nabla_{\xi} \eta, \xi\right)=2 \mathbf{g}([\xi, \eta], \xi) .
$$

Hence, $\operatorname{div} \eta=-\operatorname{tr} \operatorname{ad}_{\eta}=0$, by unimodularity of $\mathbf{G}$, and for any $\mathbf{G}$-invariant vector field $X$ follows: $\operatorname{div}(X)=\operatorname{div}(\mathscr{H}(X))$.

For $T=V \vee W$ Eq. (12) yields:

$$
\begin{aligned}
\hat{\mathcal{Q}}(f)=-\frac{1}{2}[ & \nabla_{V} \nabla_{W}+\nabla_{W} \nabla_{V}+(\operatorname{div} V) \nabla_{W}+(\operatorname{div} W) \nabla_{V}+\frac{1}{2} \operatorname{div} V \operatorname{div} W \\
& \left.+\frac{1}{4}\left((W \operatorname{div} V+V \operatorname{div} W)+\operatorname{div}\left(\nabla_{V} W\right)+\operatorname{div}\left(\nabla_{W} V\right)\right)\right] .
\end{aligned}
$$

Choosing $V=\eta$ for some invariant vertical vector field $\eta$, and an invariant vector field $W$, we get for any $\mathbf{G}$-invariant function $\phi$ :

$$
\hat{\mathcal{Q}}(f) \phi=\frac{1}{2} \operatorname{div}\left(\nabla_{\eta} W+\nabla_{W} \eta\right) \phi=\frac{1}{2} \operatorname{div}\left(2 \nabla_{\eta} W-[\eta, W]\right) \phi=\operatorname{div}\left(\nabla_{\eta} W\right) \phi,
$$

as $[\eta, W]$ is an invariant vertical vector field and hence has vanishing divergence.

In particular, if $W=\xi$ for an invariant vertical vector field $\xi, f$ is a strongly admissible polynomial corresponding to the function $\tilde{f} \equiv 0$ on $T^{*} Q$. Therefore, $\hat{\mathscr{Z}}(f)$ must satisfy the condition $\hat{\mathcal{Q}}(f) \phi=0$, i.e., $\operatorname{div}\left(\nabla_{\xi} \eta\right)=\operatorname{div}\left(\mathscr{H}\left(\nabla_{\xi} \eta\right)\right)=0$ for all invariant vertical vector fields $\xi, \eta$ and all invariant functions $\phi \in \mathscr{F}(P)$. For any invariant function $g, g \xi$ is again an invariant vertical vector field with

$$
\operatorname{div}\left(\mathscr{H}\left(\nabla_{g \xi} \eta\right)\right)=\operatorname{gdiv}\left(\mathscr{H}\left(\nabla_{\xi} \eta\right)\right)+\left(\mathscr{H}\left(\nabla_{\xi} \eta\right)\right) g,
$$

which must vanish. Hence, necessarily $\mathscr{H}\left(\nabla_{\xi} \eta\right)=0$, so the orbits must be totally geodesic submanifolds of $P$, and the first part of Theorem 3 is proved.

Choosing $W=\tilde{X}^{h}$, the horizontal lift of a vector field $\tilde{X}$ on $Q$, in (17), $f$ is an invariant function corresponding to $\tilde{f} \equiv 0$ on $T^{*} Q$. If the Dirac equivalence condition shall hold for invariant polynomials, $\hat{\mathscr{Q}}(f) \phi=0$ for any G-invariant 
$\phi \in \mathscr{F}(P)$, yielding $\operatorname{div}\left(\nabla_{\eta} \tilde{X}^{h}\right)=0$ for all $\eta$. Hence, in analogy to the preceding argument, $\nabla_{\eta} \tilde{X}^{h}$ has to be vertical for any $\eta$, yielding by (13):

$$
0=2 g\left(\nabla_{\eta} \tilde{X}^{h}, \tilde{Y}^{h}\right)=g\left(\left[\tilde{Y}^{h}, \tilde{X}^{h}\right], \eta\right),
$$

i.e., the horizontal distribution must be integrable and hence, the connection on $P$ has to be flat. The statement about the triviality of $P$ for simply connected $Q$ now simply follows from [9, Corollary 9.2].

In order to prove the rest of the theorems we need the following lemma:

Lemma 5. For any G-invariant metric on $P$, the following statements hold:

1) For any horizontal vector $X_{q} \in \mathscr{H}_{q} P$, the corresponding geodesic $\gamma(t)$ with $\dot{\gamma}(0)=X_{q}$ is horizontal and projects onto a geodesic on $Q$.

2) For arbitrary $\phi \in \mathscr{F}(Q)$ : $\left.\exp _{q}^{*}\left(\pi^{*} \phi\right)\right|_{\mathscr{H}_{q}}=\left.\left(T_{q} \pi\right)^{*}\left(\exp _{\pi(q)}^{*} \phi\right)\right|_{\mathscr{H}_{q}}$.

If the orbits are totally geodesic and the connection $\alpha$ on $P$ is flat, the following stronger statements hold:

1') Any geodesic on $P$ projects onto a geodesic on $Q$.

$\left.2^{\prime}\right)$ For arbitrary $\phi \in \mathscr{F}(Q)$ : $\exp _{q}^{*}\left(\pi^{*} \phi\right)=\left(T_{q} \pi\right)^{*}\left(\exp _{\pi(q)}^{*} \phi\right)$.

Proof of the Lemma. For any geodesic $\gamma$ there is a continuation of the vector field $\dot{\gamma}$ over $\gamma$ to a G-invariant local vector field. Using (13), it is easy to compute that $\mathbf{g}\left(\dot{\gamma}, A^{*}\right)$ is constant along any geodesic for any $A \in \mathscr{G}$. Thus, a geodesic with horizontal $\dot{\gamma}(0)$ will stay horizontal. Now, using again (13) and $\mathscr{H}\left(\left[\tilde{X}^{h}, \tilde{Y}^{h}\right]\right)=[\tilde{X}, \tilde{Y}]^{h}$ for any $\tilde{X}, \tilde{Y} \in \mathscr{X}(Q)$, one gets:

$$
(\tilde{\nabla} \tilde{X} \tilde{Y})^{h}=\mathscr{H}\left(\nabla_{X^{h}} \tilde{Y}^{h}\right) .
$$

For $\bar{\gamma}:=\pi \circ \gamma$ follows: $\left(\tilde{\nabla}_{\dot{\dot{\gamma}}}(t) \dot{\tilde{\gamma}}(t)\right)^{h}=\mathscr{H}\left(\nabla_{\dot{\forall}(t)} \dot{\gamma}(t)\right)=0$, i.e., the projection $\tilde{\gamma}$ of $\gamma$ is a geodesic. Now, statement 2) follows immediately from $\left.\pi \circ \exp _{q}\right|_{\mathscr{H}_{q}}$ $=\left.\exp _{\pi(q)} \circ T_{q} \pi\right|_{\mathscr{H}_{q}}$ which is an immediate consequence of statement 1).

If the orbits are totally geodesic and the connection $\alpha$ on $P$ is flat, then $\nabla_{\eta} \tilde{X}^{h}$ and $\nabla_{\eta} \xi$ are vertical for arbitrary $\tilde{X} \in \mathscr{X}(Q)$ and arbitrary vertical vector fields $\xi, \eta$, yielding:

$$
\mathscr{H}\left(\nabla_{Y} X\right)=\mathscr{H}\left(\nabla_{\mathscr{H} Y} \mathscr{H} X\right)
$$

for any $\mathbf{G}$-invariant vector fields $X, Y$. This implies, again by $\mathbf{G}$-invariant continuation of the vector field $\dot{\gamma}$ over $\gamma$, that the projection of any geodesic to $Q$ coincides with the projection of the horizontal geodesic $\hat{\gamma}$ with $\dot{\hat{\gamma}}(0)=\mathscr{H}(\dot{\gamma}(0))$, so it is a geodesic.

Statement $2^{\prime}$ ) follows from $\pi \circ \exp _{q}=\exp _{\pi(q)}{ }^{\circ} T_{q} \pi$ which is again an immediate consequence of statement $1^{\prime}$ ).

Now choose a local trivialization of the bundle $P$ and adapted coordinates $\left(\phi_{\alpha}, \tilde{q}_{\mu}\right)$. As $\mathbf{g}$ is block-diagonal for a frame consisting of vertical and horizontal vectors and the coordinate frames adapted to a local trivialization differ from such frames by a matrix with determinant one, we get:

$\int d \phi_{1} \ldots d \phi_{r} \sqrt{\operatorname{det} \mathbf{g}}=\int d \phi_{1} \ldots d \phi_{r} \sqrt{\operatorname{det}\left(\mathbf{g}_{\alpha \beta}\right)_{\alpha, \beta=1} \ldots r} \sqrt{\operatorname{det} \tilde{\mathbf{g}}(\tilde{q})}=\kappa \sqrt{\operatorname{det} \tilde{\mathbf{g}}(\tilde{q})}$.

Let the orbits of $\mathbf{G}$ on $P$ be totally geodesic and the connection form $\alpha$ be flat. Set $\phi=U \widetilde{\phi}, \psi=U \widetilde{\psi}$ for $\widetilde{\phi}, \widetilde{\psi} \in \hat{\mathbf{H}}_{\text {int }}$. Using the lemma and the constancy of 
$\kappa$ following from the geodesic completeness of the orbits we get for arbitrary $a \in T_{x} P$ :

$$
\begin{aligned}
\left.\frac{d^{k}}{d \lambda^{k}} D(\widehat{x, \lambda} a)\right|_{\lambda=0} & =\left.\frac{d^{k}}{d \lambda^{k}} \chi(x, \lambda a)\left\{\left(\exp _{x}^{*} \psi\right) \overline{\left(\left(\exp _{x} \circ I_{x}\right)^{*} \phi\right)}\right\}(\lambda a)\right|_{\lambda=0} \\
& =\left.\kappa^{-1} \frac{d^{k}}{d \lambda^{k}}\left[\left(T_{x} \pi\right)^{*}\left\{\left(\exp _{\tilde{x}}^{*} \tilde{\psi}\right) \overline{\left(\left(\exp _{\tilde{x}} I_{\tilde{x}}\right)^{*} \tilde{\phi}\right)}\right\}(\lambda a)\right]\right|_{\lambda=0} \\
& =\kappa^{-1} \pi *\left\{\left.\frac{d^{k}}{d \lambda^{k}} \tilde{D}\left(\tilde{x}, \hat{\lambda} \widehat{T_{x}} \pi(a)\right)\right|_{\hat{\lambda}=0}\right\}
\end{aligned}
$$

As any operator $O$ of the form $O f=\left.\frac{\hat{O}^{k}}{\partial v^{i_{1}} \ldots \partial v^{i_{r}}} f\right|_{v=0}$ may be obtained as a linear combination of operators of the form $\left.\frac{d^{k}}{d \lambda^{k}} f(\lambda a)\right|_{\hat{\lambda}=0}$ for suitable vectors $a$, and as the G-invariant function $\left(X_{1} \vee \ldots \vee X_{r}\right)^{i_{1} \ldots i_{r}} p i_{1} \ldots p i_{r}$ for G-invariant vector fields $X_{1}, \ldots, X_{r}$ on $P$ corresponds via (1) to the function $\left(\pi_{*}\right.$ $\left.X_{1} \vee \ldots \vee \pi_{*} X_{r}\right)^{\mu_{1} \ldots \mu_{r}} \tilde{p}_{\mu_{1}} \ldots \tilde{p}_{\mu_{r}}$, we may conclude:

$$
\left.T^{i_{1} \ldots l_{r}} \frac{\partial^{k}}{\partial v^{i_{1}} \ldots \partial v^{i_{r}}} D(\widehat{x, v})\right|_{v=0}=\left.\kappa^{-1} \tilde{T}^{\mu_{1}} \ldots \mu_{r} \frac{\partial^{k}}{\partial \tilde{v}^{\mu_{1}} \ldots \partial \tilde{v}^{\mu_{r}}} \tilde{D}(\widehat{\tilde{x}, \tilde{v}})\right|_{v=0}
$$

for any $\mathbf{G - i n v a r i a n t ~ f u n c t i o n} T^{i_{1}} \ldots i_{r} p_{i_{1}} \ldots p_{i_{r}}$ corresponding to $\tilde{T}^{\mu_{1}} \ldots \mu_{r} \tilde{p}_{\mu_{1}} \ldots \tilde{p}_{\mu_{r}}$ on $T^{*} Q$. Hence, using (18),

$$
\left\langle U \tilde{\phi}, \hat{\mathscr{Q}}_{\mathrm{ext}}\left(T^{i_{1} \ldots i_{r}} p_{i_{1}} \ldots p_{i_{r}}\right) U \tilde{\psi}\right\rangle=\left\langle\tilde{\phi}, \hat{\mathscr{Q}}_{\mathrm{int}}\left(\tilde{T}^{\mu_{1}} \ldots \mu_{r} \tilde{p}_{\mu_{1}} \ldots \tilde{p}_{\mu_{r}}\right) \tilde{\psi}\right\rangle
$$

and Theorem 4 is proved.

If we drop the assumption that the connection form $\alpha$ is flat, the argumentation above goes through as long as one restricts oneself to vectors $a$ which are horizontal. Hence, one can prove in a completely analogous way, that for any "horizontal" G-invariant function (20) still holds. On the other hand, as $\phi, \psi$ are constant along the orbits and as the curves $\exp (\lambda a)$ for vertical $a$ do not leave the orbit because the orbits are totally geodesic, $\left.\frac{d^{k}}{d \lambda^{k}} D(\widehat{x, \lambda} a)\right|_{\lambda=0}=0$ for vertical $a$. Thus any "vertical" function is quantized by an operator that vanishes on $\hat{\mathbf{H}}_{\text {phys }}$ and we may conclude that the Dirac equivalence condition is satisfied for all strongly admissible functions.

Q.E.D.

Theorem 6. The Dirac equivalence condition for arbitrary "horizontal" quadratic observables holds iff the volume $\kappa$ of the orbits satisfies the equation $\tilde{\nabla} d \log (\kappa)=0$. If $Q$ is geodesically complete, this condition may be satisfied by a nonconstant volume function iff there is a global isometric diffeomorphism from $Q$ to $\mathbb{R} \times \hat{Q}$ for a Riemannian manifold $(\hat{Q}, \hat{\mathbf{g}})$, where $\mathbb{R} \times \hat{Q}$ is endowed with the product metric induced by $\hat{\mathbf{g}}$ and a constant multiple of the standard metric on $\mathbb{R}$. In particular, if $Q$ is compact, the condition is satisfied iff the orbit volume is constant.

Proof. It is sufficient to restrict oneself to functions of the form $\left(\tilde{X}^{h} \vee \tilde{Y}^{h}\right)^{i j} p_{i} p_{j}$ for $\tilde{X}, \tilde{Y} \in \mathscr{X}(Q)$, which correspond to functions $(\tilde{X} \vee \tilde{Y})^{\mu v} \tilde{p}_{\mu} \tilde{p}_{v}$ on $T^{*} Q$. Using

$$
\operatorname{div}\left(\tilde{X}^{h}\right)=\pi^{*}\left(\widetilde{\operatorname{div}}(\tilde{X})+\frac{\tilde{X} \cdot \kappa}{\kappa}\right), \quad \mathscr{H}\left(\nabla_{\tilde{X}^{h}} \tilde{Y}^{h}\right)=(\tilde{\nabla} \tilde{X} \tilde{Y})^{h}, \quad \mathscr{V}(X) \cdot h=0
$$


for any $\tilde{X} \in \mathscr{X}(Q), X \in \mathscr{X}(P)$ and any $\mathbf{G}$-invariant $h$, and the explicit form (15) of the isomorphism of $\hat{\mathbf{H}}_{\text {phys }} \subset \hat{\mathbf{H}}_{\text {ext }}$ and $\hat{\mathbf{H}}_{\text {int }}$, a direct computation yields:

$$
\begin{gathered}
\left(U^{-1} \hat{\mathscr{Q}}_{\mathrm{ext}}\left(\left(\tilde{X}^{h} \vee \tilde{Y}^{h}\right)^{i j} p_{i} p_{j}\right) U-\hat{\mathscr{Q}}_{\mathrm{int}}\left((\tilde{X} \vee \tilde{Y})^{\mu v} \tilde{p}_{\mu} \tilde{p}_{v}\right)\right) \phi_{\mathrm{int}} \\
=\frac{1}{8}\left(2 \frac{(\tilde{X} \kappa)(\tilde{Y} \kappa)}{\kappa^{2}}-\frac{\tilde{X}(\tilde{Y} \kappa)}{\kappa}-\frac{\tilde{Y}(\tilde{X} \kappa)}{\kappa}+\frac{\left(\tilde{\nabla}_{\tilde{Y}} \tilde{X}\right) \kappa}{\kappa}+\frac{\left(\tilde{\nabla}_{\tilde{X}} \tilde{Y}\right) \kappa}{\kappa}\right) \phi_{\text {int }} .
\end{gathered}
$$

The difference term, that has to vanish, is symmetric and bilinear in $\tilde{X}, \tilde{Y}$. Hence, by a polarization argument, it vanishes for all $\tilde{X}, \tilde{Y} \in \mathscr{X}(Q)$ iff it vanishes for $\tilde{X}=\tilde{Y}$, i.e., iff

$$
\frac{(\tilde{X} \kappa)^{2}}{\kappa^{2}}-\frac{\tilde{X}(\tilde{X} \kappa)}{\kappa}+\frac{(\tilde{\nabla} \tilde{X} \tilde{X}) \kappa}{\kappa}=0
$$

Setting $\kappa=e^{\rho}$ for some function $\rho$ yields in local coordinates: $\tilde{X}^{\mu} \tilde{X}^{v} \partial_{\mu} \partial_{\nu} \rho-\tilde{X}^{\mu} \tilde{X}^{v} \Gamma_{\mu \nu}^{\omega} \partial_{\omega} \rho=0$, hence, as $\tilde{X}$ is arbitrary and the connection has no torsion: $\tilde{\nabla} d \rho=0$.

Now, assume that $Q$ is geodesically complete and that there is a nonconstant function $\rho=\log (\kappa)$ satisfying $\tilde{\nabla} d \rho=0$. Define a vector field $\tilde{X}$ on $Q$ by $\tilde{X}=g^{\sharp}(d \rho)$. Then, $\tilde{\nabla} \tilde{X}=0$, and in particular, if there is any point where $\tilde{X}$ does not vanish (i.e., if $\rho$ is not constant), then $\tilde{X}$ vanishes nowhere. As $\tilde{\nabla}_{\tilde{X}} \tilde{X}=0$ by $\tilde{\nabla} d \rho=0$ and as $Q$ is assumed to be geodesically complete, the vector field $\tilde{X}$ is complete. Since

$$
\mathbf{g}(\tilde{X}, \tilde{X})=\mathbf{g}^{-1}(d \rho, d \rho)=\text { const. } \neq 0 .
$$

the surfaces of constant $\rho$ are diffeomorphic submanifolds of $Q$, the diffeomorphisms being just given by the flow of $\tilde{X}$. As $\rho$ increases monotonically along the flow lines of $\tilde{X}, Q$ is indeed diffeomorphic to $\mathbb{R} \times \hat{Q}$, where $\hat{Q}$ is $\rho^{-1}(c)$ for some $c$ in the range of $\rho$. ( $Q$ is connected, as $P$ is connected, hence the diffeomorphism defined in this way is indeed onto.)

Choosing local coordinates $\left(r, \hat{q}^{\alpha}\right)$ on $\mathbb{R} \times \hat{Q}$, the vector field $\tilde{X}$ is mapped to $\frac{\partial}{\partial r}$. As $\tilde{X}=\mathbf{g}^{\sharp}(d \rho)$ is orthogonal to $\rho^{-1}(c)$ for any $c \in \mathbb{R}$, and $\tilde{\nabla} \tilde{X}=0$, one easily computes that the metric on $\mathbb{R} \times \hat{Q}$ has to be of the form $a d r^{2}+\hat{\mathbf{g}}^{\alpha \beta}(\hat{q}) d \hat{q}^{\alpha} d \hat{q}^{\beta}$ for some $a \in \mathbb{R}$ and some Riemannian metric $\hat{\mathbf{g}}$ on $\hat{Q}$.

On the other hand, if $\mathbb{R} \times \hat{Q}$ is endowed with a metric of this form, any function of the form $a r+b(a, b \in \mathbb{R})$ satisfies $\tilde{\nabla} d(a r+b)=0$.

If $Q$ is compact, then it is geodesically complete for any Riemannian metric on $Q$, but certainly not diffeomorphic to $\mathbb{R} \times \hat{Q}$ for any $\hat{Q}$. Hence, the last statement follows.

Q.E.D

\section{Remarks.}

1. For the construction of the diffeomorphism only the completeness of the special vector field $\tilde{X}$ is needed, the geodesic completeness is only used to guarantee the completeness of $\tilde{X}$.

2. For a generic metric $\tilde{\mathbf{g}}$ on $Q$, there are no Killing fields on $Q$. On the other hand, any covariant constant vector field is a Killing field. Hence, for a generic metric $\tilde{\mathbf{g}}$ on $Q, \kappa$ has to be constant. 
3. Even if the vector field $\tilde{X}$ is not complete, $Q$ is locally diffeomorphic to $\mathbb{R} \times \hat{Q}$ in an open neighbourhood of any $\kappa^{-1}(c)$.

\section{Weyl Ordering for Half-Densities}

In the previous section we studied the equivalence problem for the modified Weyl ordering method, which uses a fixed measure instead of half-densities. Although there is no criterion by which one might a priori decide which quantization scheme is "better", as different quantum theories may have the same classical limit, the original version of the generalized Weyl ordering due to Underhill might seem more appealing, as it is closer to usual geometric quantization. However, if one studies the equivalence problem using this quantization scheme for extrinsic and intrinsic quantization, the situation is much worse than for the ansatz studied in the previous section:

The additional problems arise already for quadratic observables because of the curvature term in (9). In general, the components of the Ricci-tensor on $P$ are quite complicated functions of the metric $\tilde{\mathbf{g}}$ on $Q$, the metrics $\mathbf{h}$ on the orbits and the connection $\alpha[2,3]$. Even in the nicest case, when $P$ is a trivial bundle over $Q$, the connection $\alpha$ is flat, and $\mathbf{h}$ is a constant metric, the vertical components of the Ricci tensor of $\mathbf{g}$ do not vanish but are just the corresponding components of the Ricci tensor of $\mathbf{h}$ on the respective orbit.

Hence, as the other terms in (9) vanish on $\mathbf{H}_{\mathrm{phys}}$ in analogy to the preceding section for an invariant "vertical" function $f=(\eta \vee \xi)^{i j} p_{i} p_{j}, \mathscr{Q}_{\text {ext }}(f)$ acts on $\mathbf{H}_{\text {phys }}$ by multiplication with $-\frac{1}{12}(\eta \vee \xi)^{i j} R_{i j}$. Now, since a generic Lie group does not admit a metric with vanishing curvature, the Dirac equivalence condition may not even be satisfied for all strongly admissible quadratic functions. Thus, if one considers the original quantization scheme using half-densities superior to the modified ansatz using functions square integrable with respect to $\mu$, one would like to take it for the reduced quantization. (We are assuming that the symmetry is a "redundancy symmetry"!) Hence, we have to modify the extrinsic quantization scheme appropriately in order to ensure at least the validity of theorems analogous to those of the preceding section. Essentially, we have to find a possibility of treating the "horizontal directions" of a half-density as in the original quantization scheme, the "vertical directions" however as in the modified scheme. This is possible, indeed:

To this end, we define an appropriate map $\tau_{q}$ mapping a half-density on $P$ to half-densities on suitable open neighbourhoods of 0 in $T_{q} P$ for arbitrary $q \in P$. (In the original scheme, this was just the pull-back via exp, whereas in the modified scheme this mapping was given by $\phi|\mu|^{\frac{1}{2}} \mapsto\left(\exp ^{*} \phi\right)\left|d^{n} v(q)\right|^{\frac{1}{2}}$.) As $\pi \circ \exp _{q}$ restricted to $\mathscr{H}_{q} P$ is a local diffeomorphism from $\mathscr{H}_{q} P$ to $Q$, we can pull back the canonical half density $|\tilde{\mu}|^{\frac{1}{2}}$ on $Q$ induced by $\tilde{\mathbf{g}}$ to an open neighbourhood $U$ of 0 in $\mathscr{H}_{q} P$, yielding a (non-constant) half-density $\rho_{\mathscr{H}}$ on $U \subset \mathscr{H}_{q} P$. On the other hand, we may define a constant half-density $\rho_{\mathscr{Y}}$ on the vertical space $\mathscr{V}_{q} P$ with the help of the Euclidean metric on the vector space $\mathscr{V}_{q} P$ induced by $\mathbf{h}$. Now, we can define a half-density $\rho_{q}$ on $U \times \mathscr{V}_{q} \subset T_{q} P$ as the "product" of $\rho_{\mathscr{H}}$ and $\rho_{\mathscr{r}}$, i.e., we set

$$
\rho_{q}\left(V_{1}, \ldots, V_{r}, X_{1}, \ldots, X_{n-r}\right)=\rho_{\mathscr{H}}\left(X_{1}, \ldots, X_{n-r}\right) \rho_{\mathscr{T}}\left(V_{1}, \ldots, V_{r}\right)
$$


for special frames consisting only of vertical vectors $V_{i}$ and horizontal vectors $X_{i}$. On arbitrary frames $\xi$ we may define $\rho_{q}$ by the property of half-densities: $\rho_{q}(\underline{\xi} \cdot g)=\sqrt{|g|} \rho_{q}(\xi)$ for $g \in G L(n, \mathbb{R})$.

Hence, we may set: $\tau_{q}\left(\hat{\phi}|\mu|^{\frac{1}{2}}\right):=\left(\exp ^{*} \hat{\phi}\right) \rho_{q}$ for $\hat{\phi} \in \mathscr{F}(P)$. In the extreme cases that the orbits are either zero or $n$ dimensional the resulting mapping coincides with that for the unmodified quantization scheme using half-densities in the first and with the modified scheme using functions in the latter case. Using that function, we can again define for $\phi, \psi \in \mathbf{H}_{\mathrm{ext}}$ a function $\Phi\left(\alpha_{q}\right)$ as:

$$
\Phi\left(\alpha_{q}\right):=\int e^{-2 i \alpha_{q}\left(v_{q}\right)} \chi\left(q, v_{q}\right)\left(\tau_{q} \psi\right) \overline{\left(\left(I_{q}\right)^{*} \tau_{q} \phi\right)},
$$

and the operator $\mathscr{Z}(f)$ to a function $f$ by (6) with the new $\Phi\left(\alpha_{q}\right) \cdot \mathscr{2}(f)$ can be again easily seen to be a differential operator of the order of the degree of the polynomial $f$, and to be formally selfadjoint (by the essentially symmetric appearance of $\phi, \psi$ in (21)).

For this quantization scheme, one can compute that quadratic functions $T^{i j} p_{i} p_{j}$ are quantized by the operator (9) with the curvature term replaced by

$$
\frac{1}{12}\left(T_{q} \pi T(q)\right)^{\mu \nu} \tilde{R}_{\mu v}(\pi(q))
$$

Hence, it is obvious that with the new extrinsic quantization scheme, and the unmodified Underhill quantization scheme for the intrinsic quantization, just the same necessary conditions for the validity of the Dirac equivalence condition for strongly admissible, arbitrary and "horizontal" invariant quadratic functions follow as in the last section. Furthermore, the proofs of the corresponding theorems may be easily modified for this method, and Theorems 3, 4 and 6 may be seen to hold with the new ansatz as well.

Remark. It might seem geometrically more natural to try to achieve the necessary, different treatment of the horizontal and vertical directions of the half-densities by choosing a fixed "vertical half-density" on $P$, using this to map a half-density on $P$ to a "horizontal half-density" on $P$ by writing it as a product of this "horizontal half-density" and the fixed "vertical half-density", and pulling it back to $T_{q} P$ via exp. This can be done in a completely well-defined way, indeed. However, as two vectors in $T_{q} P$ differing by a vertical vector are generally not mapped to the same vector by $T \exp _{q}$, there would arise additional terms already in the quantization of quadratic functions containing in particular the curvature of the connection $\alpha$. Hence, the equivalence even for strongly admissible quadratic functions would only hold if the connection $\alpha$ is flat.

\section{Conclusions}

In the preceding two sections we have proved equivalence theorems for the extrinsic and intrinsic quantization of various classes of functions. Theorem 4 is negative in character, as it asserts that extrinsic and intrinsic quantization for arbitrary invariant functions can be equivalent essentially only on trivial bundles. As, to the contrary, any principal bundle admits for any metric $\tilde{\mathbf{g}}$ on the base manifold $Q$ a compatible metric for which the orbits are totally geodesic, 
Theorem 3 ensures that for a restricted class of functions one may always obtain equivalence by a proper choice of the metric $\mathbf{g}$ on $P$.

However, if one is interested in generalizations to cases where the isotropy groups are not trivial and not conjugated, even Theorems 3 and 6 take a negative character: Already when the generic isotropy groups are trivial, but some points have nontrivial isotropy groups, the function $\kappa$ is zero on those points for any smooth metric $\mathbf{g}$, in contradiction to the needed constancy of $\kappa$. Hence, even for the extrinsic quantization, where the configuration space is smooth, those points have to be cut out, resulting in the necessity of studying boundary conditions and selfadjoint extensions. Hence, the ambiguities arising in intrinsic quantization due to the singular structure of configuration space [7], cannot be remedied by using a formally equivalent extrinsic quantization on the smooth extrinsic configuration space.

Now, the question naturally arises, to what extent our results depend on the particular quantization scheme. The main problem in answering that question is that there seem to be no other general quantization schemes apart from BKSkernels which assign uniquely defined operators on some Hilbert-space to a sufficiently large class of functions. (Using a different polarization in geometric quantization only shifts the class of polarization preserving functions without sufficiently enlarging it; star product quantization yields concrete star products only in particular examples.) For the BKS-procedure the situation is expected to be even worse: First, the class of functions quantizable in this way is rather obscure due to the complicated regularity conditions necessary for its application and mainly due to the fact that there is no general criterion to decide whether the constructed operator will even be formally selfadjoint [15]. Secondly, this ansatz does not have the property of mapping the sum of functions to the sum of the corresponding operators. Hence, even if "vertical" polynomials are quantized by operators vanishing on $\mathbf{H}_{\text {phys }}$ and the equivalence condition holds for a "horizontal" polynomial, it is far from obvious whether this will still hold for the sum of the two.

This non-additivity property will be shared by any quantization scheme, which has the property of quantizing every polynomial of the form $T^{i j} p_{i} p_{j}$ for nondegenerate $T^{i j}$ by the corresponding Laplacian if the curvature of $T_{i j}$ vanishes. This problem is avoided by the quantization scheme studied by us, because it quantizes $T^{i j} p_{i} p_{j}$ only by the Laplacian if the chosen connection $\nabla$ is the Levi-Civita connection to $T^{i j}$.

This, and the additional problems encountered for the unmodified quantization scheme using half-densities, may be considered as a very strong hint that the situation dealt with in Sect. 4 is the optimal one for any "natural" extrinsic quantization scheme, not modified "by hand" to give a better agreement between extrinsic and intrinsic quantization.

On the other hand, as we have seen in Sect. 5, one may always get rather strong equivalence results if one modifies the extrinsic quantization scheme "by hand," using a splitting into "horizontal" and "vertical" directions and treating both asymmetrically. The most extreme such possibility would be to define the quantization of "horizontal" polynomials by just pulling back the corresponding intrinsic operator via the isomorphism of $\mathbf{H}_{\text {int }}$ and $\mathbf{H}_{\text {phys }}$ (defining it to be zero on the orthogonal complement of $\mathbf{H}_{\text {phys }}$ in $\mathbf{H}_{\text {ext }}$ ), and to quantize any "vertical" polynomial in such a way that it vanishes on $\mathbf{H}_{\text {phys }}$, for example by using standard or antistandard ordering. Such an ansatz would be very much in the spirit of [10]. However, if one is really interested in dealing with redundancy symmetries (for real 
physical symmetries, relating physically different states, the equivalence question is insignificant anyway, as in this case the intrinsic ansatz has no physical meaning), the question arises whether such a modified ansatz is still of any value: The two main advantages of extrinsic quantization mentioned in the introduction are both of purely practical, computational character: They were the greater simplicity of extrinsic configuration space in many applications and the greater ease of maintaining manifest Lorentz covariance in relativistic theories. Both advantages will obviously be spoilt by any ansatz needing a detailed splitting into horizontal and vertical directions and an asymmetric treatment of both.

Acknowledgement. I should like to thank Th. Filk and H. Römer for helpful discussions, and W. Bischoff for the careful reading of the manuscript.

\section{References}

1. Ashtekar, A., Stillerman, M.: Geometric quantization and constrained systems. J. Math. Phys. 27, 1319-1330 (1986)

2. Besse, A.L.: Einstein manifolds. Springer 1987

3. Coquereaux, R., Jadczyk, A.: Riemannian Geometry, Fiber Bundles. Kaluza-Klein Theories and All That .... Singapore: World Scientific 1988

4. Dirac, P.A.M.: Lectures on Quantum mechanics, Belfer Graduate School of Science Monograph Series 2 (1964)

5. Duval, C., Elhadad, J., Gotay, M. J., Śniatycki, J., Tuynman, G.M.: Geometric quantization and bosonic BRST theory. Ann. Phys. 206, 1-26 (1991)

6. Duval, C., Elhadad, J., Tuynman, G.M.: The BRS method and geometric quantization: Some examples. Commun. Math. Phys. 126, 535-557 (1990)

7. Emmrich, C., Römer, H.: Orbifolds as configuration spaces of systems with gauge symmetry. Commun. Math. Phys. 129, 69-94 (1990)

8. Gotay, M.J.: Constraints, reduction, and quantization. J. Math. Phys. 27, 2051-2066 (1986)

9. Kobayashi, S., Nomizu, K.: Foundations of Differential Geometry. Inter-science Publishers 1963

10. Kuchar̆, K.: Covariant factor ordering of gauge systems. Phys. Rev. D 34, 3044-3057 (1986)

11. Kummer, M.: On the construction of the reduced phase space of a Hamiltonian System with symmetry. Indiana Univ. Math. J. 30, 281-291 (1981)

12. Liu Zhang-Ju, Qian Min,: Gauge invariant quantization on Riemannian manfolds. Trans. Am. Math. Soc. 331, 321-333 (1992)

13. Loll, R.: Noncommutativity of constraining and quantizing: A U(1)-gauge model. Phys. Rev. D, 41, 3785-3791 (1990)

14. Romano, J.D., Tate, R.S.: Dirac versus reduced space quantisation of simple constrained systems. Class Quant. Grav. 6, 1487-1500 (1989)

15. Śniatycki, J.: Geometric Quantization and Quantum Mechanics. Applied Mathematical Sciences 30, Springer 1980

16. Tuynman, G.M.: Reduction, quantization, and nonunimodular groups. J. Math. Phys. 31, 83-90 (1990)

17. Tuynman, G.M.: Quantization of first class constraints with structure functions. CNRS Luminy preprint CPT-90/P.2401

18. Underhill, J.: Quantization on a manifold with connection. J. Math. Phys. 19, 1932-1935 (1978)

19. Widom, H.: A Szego theorem and symbol calculus for pseudodifferential operators. Ann. of Math. Stud. 91, Princeton University Press (1979), 261-283

20. Widom, H.: Families of Pseudodifferential Operators. In: Topics in Functional Analysis (dedicated to M.G. Krein) Gohberg, I., Kac, M. (eds.) New York: Academic Press 1978 